\title{
La presentación de un Proyecto Europeo como herramienta docente. Más allá de las aulas
}

\author{
Juan V. Oltra Gutiérrez ${ }^{\text {a }}$, Hermenegildo Gil Gómez ${ }^{\text {b }}$, Raúl Oltra Badenes ${ }^{c}$ \\ Departamento de Organización de Empresas. Universitat Politècnica de València, España, \\ jvoltra@doe.upv.es ${ }^{\mathrm{a}}$; hgilgom@doe.upv.es ${ }^{\mathrm{b}}$; rauloltra@doe.upv.es ${ }^{\mathrm{c}}$
}

\begin{abstract}
Resumen
En la presente comunicación se expone una experiencia docente, centrada en la aproximación de una actividad de investigación, focalizada en los contenidos de una asignatura concreta, a alumnos de grado. El objetivo último es estimular su curiosidad e inducirles a profundizar en la materia expuesta. Para conseguirlo, se realiza una presentación a la que opcionalmente pueden acudir los alumnos y en base a ella se prepara una actividad incardinada en la Gamificación efectuada en la asignatura.
\end{abstract}

Palabras clave: Gamificación; Actividades voluntarias; Docencia.

\begin{abstract}
In the present communication a teaching experience is exposed, focused on the approximation of a research activity, focused on the contents of a specific subject, to undergraduate students. The ultimate goal is to stimulate their curiosity and induce them to delve into the exposed matter. To achieve this, a presentation is made to which students can optionally attend and based on it an activity incardinated in the Gamification carried out in the subject is prepared.
\end{abstract}

Keywords: Gamification; Voluntary activities; Teaching.

\section{Introducción}

Muchas veces a los docentes nos queda una duda: ¿hemos sabido transmitir la importancia de los contenidos de nuestras asignaturas? O, por expresarlo de forma más prosaica: ¿nuestros alumnos se han dado cuenta de que lo que contamos en clase tiene sentido una vez tengan el título en el bolsillo? 
Si esta pregunta se repite en materias con una aplicación más o menos evidente, como puede ser algorítmica o programación en la carrera de informática, es obvio que la preocupación crece cuando se trata de materias de corte jurídico, donde el conocimiento de la normativa se ve a priori más como un castigo que como algo útil.

En el caso que nos ocupa, tratamos de incrementar la curiosidad de los alumnos, y con ello el aprecio por las materias que están recibiendo, mediante la exposición de un proyecto europeo, cuyo fundamento reside en buena parte en los conocimientos que forman parte del temario de la asignatura.

En el presente trabajo describiremos, en el apartado "Objetivos" la asignatura en cuestión donde la experiencia es llevada a cabo y las líneas generales del proyecto que se les expone para centrar que es lo que intentamos conseguir; en el apartado "Desarrollo de la experiencia" la experiencia en sí misma: la logística de la presentación y las actividades ligadas a la misma y en "Resultados" los elementos cuantificables de la misma, así como los efectos que subjetivamente han podido recogerse.

\section{Objetivos}

La experiencia se desarrolla en los siete grupos de segundo semestre de la asignatura Deontología y Profesionalismo perteneciente al grado en Informática, durante el primer semestre, código 11550, en adelante DyP. Esta asignatura tiene otro grupo en el primer semestre, dentro del doble grado grado de Informática y Administración de Empresas. (Oltra Gutiérrez, Método del caso como medio de evaluación dentro del modelo FLIPTeaching. Una experiencia con una asignatura de corte jurídico para ingenieros., 2018). Las características principales de la asignatura pueden verse en la tabla 1.

Tabla 1. Características de la asignatura.

\begin{tabular}{ll}
\hline Asignatura / características & DyP \\
\hline Créditos totales de teoría & 3 \\
\hline Créditos totales de práctica & 1,5 \\
\hline Inicio de clases & $29 /$ enero \\
\hline Fin de clases & $1 /$ junio \\
$\mathbf{N}^{\mathbf{0}}$ de clases de teoría en el semestre & $14-16^{*}$ \\
$\mathbf{N}^{\mathbf{0}}$ de actividades distintas de gamificación realizadas & 3 \\
\hline
\end{tabular}

* Depende del calendario de cada grupo. Fuente: Elaboración propia 
Sus contenidos son de marcado tipo legal. Tras presentar los marcos profesionales vigentes en Europa, se les presenta la normativa legal más relevante para su profesión, tanto española como de la Unión, para acabar con un apartado dedicado a la deontología profesional en informática.

Buscamos un objetivo: estimular su curiosidad e inducirles a profundizar en la materia expuesta.

Para conseguirlo se les presenta la realidad de un proyecto europeo, del que el profesor responsable de la asignatura forma parte: el proyecto MADE (Multiple Access to eDElivery)

¿Qué es "MADE"? El objetivo principal de este proyecto es promover seis nuevos puntos de acceso del consorcio MADE, integrando el protocolo AS4 y desarrollando sus Service Metadata Publishers (SMP), para el uso de eDelivery DSI (Digital Service Infrastructure) entre los sectores público y privado. En MADE participan algunas de las empresas más reconocidas especializadas en el sector (EDICOM, BlZbrains, Deficient, Aksesspunkt, eConnect y Unimaze). Se deben cumplir los requisitos establecidos en el Reglamento eIDAS (Reglamento UE 910/2014 sobre identificación electrónica y servicios de confianza). El proyecto también tendrá en cuenta el marco legal y técnico definido por las reglamentaciones y directivas europeas, especialmente la recalculo de eIDAS, para promover el uso de eDelivery DSI en toda Europa. De hecho, las tareas asignadas al equipo de la UPV responden al objetivo de analizar el contexto legal de los proveedores de servicios de confianza (TSP) que ofrecen los servicios de entrega electrónica para sus clientes a nivel nacional y europeo. Se analiza el contexto nacional de los clientes, revisando tanto las reglas legales existentes como la transcripción y aplicación esperadas de la regulación eIDAS. También incluye revisar las leyes relacionadas con la protección de datos personales. (Universidad Carlos III de Madrid, 2017)

Con ese proyecto presentado, se les invita a participar en actividades voluntarias relativas al mismo, para mejorar no solo sus conocimientos, sino su interés por la materia.

\section{Desarrollo de la experiencia}

Todos los cursos se presentan charlas invitadas, con el interés puesto en relacionar lo que en la calle se hace con lo que se cuenta en las aulas. Otros años se ha invitado a legisladores que han escrito parte de leyes que el alumno estudia, a empresas con fuerte impacto donde los ponentes explican como en el día a día precisan de los contenidos de la asignatura... la participación tradicionalmente ha rondado un $50 \%$ del alumnado. 
El 10 de mayo de 2018, en el Salón de Actos de la Escuela Técnica Superior de Informática Aplicada (ETSINF) de la UPV, se presentó el proyecto con el aforo al completo y siendo retransmitido el mismo por streaming. Estaban invitados los 369 alumnos de la asignatura DyP en particular, y en general cualquier alumno de la ETSINF y de la UPV. Se difundió de forma previa el acto, y se publicitó de qué forma los alumnos que temieran no poder entrar por falta de espacio, podían seguir el acto en directo, o una vez acabado, mediante grabación.

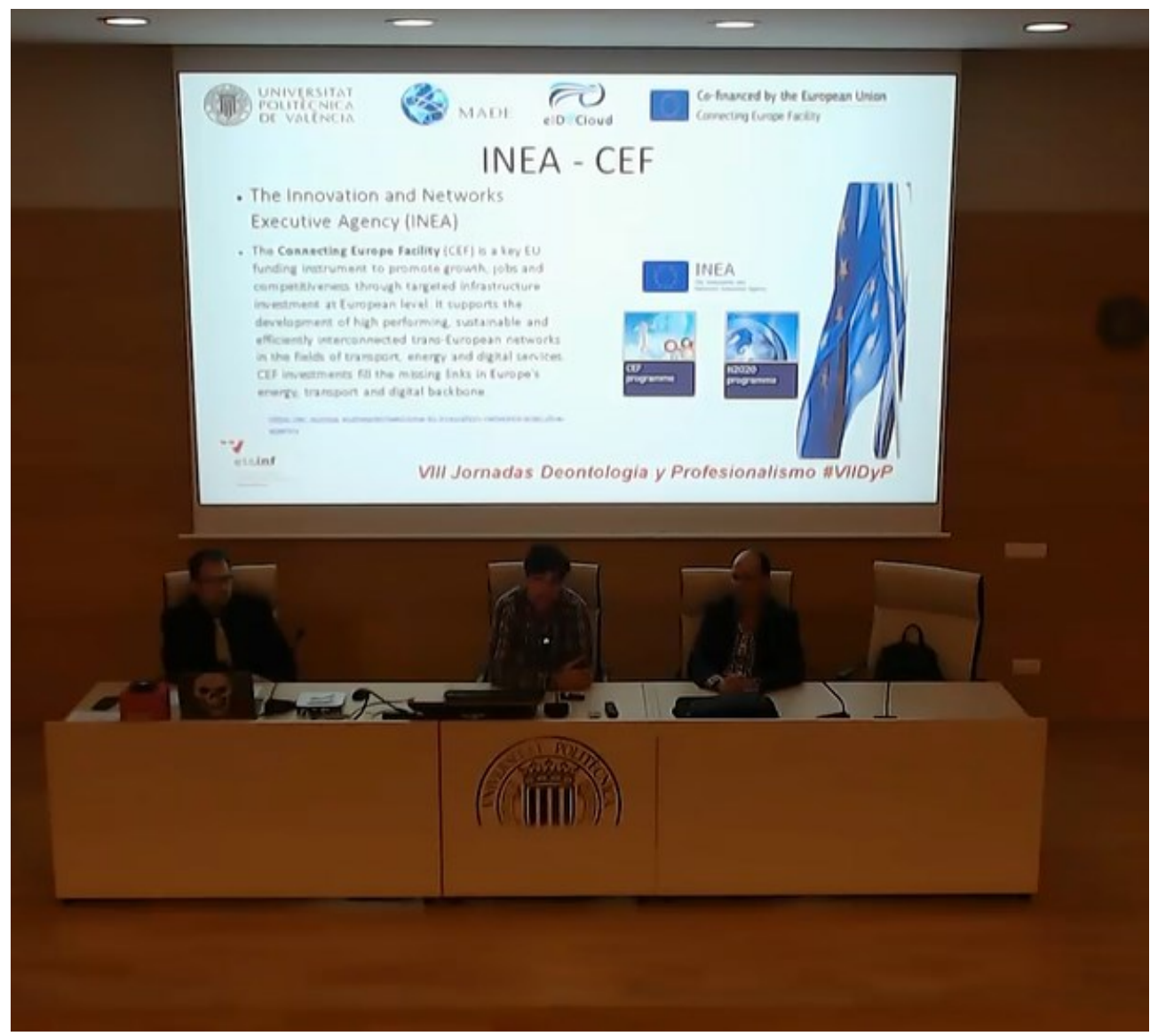

Fig. 1 Momento de la presentación. Foto: Raúl Oltra.

Mientras los ponentes introducían los conceptos a los alumnos, el moderador difundía por twitter los aspectos más relevantes, de forma que pudiera servir a posteriori de recordatorio rápido de lo dicho y, también, para que el acto tuviera mayor difusión, pues la cuenta desde la que se retransmitió la siguen alumnos de cursos precedentes. 
Los alumnos matriculados en la asignatura fueron avisados mediante la plataforma interna de la universidad de forma previa de la realización de un test sobre la misma, incardinado en las actividades de gamificación de la asignatura. (Oltra Gutiérrez, Gamificación e Informática. Comparativa de una experiencia mixta en Grado y Máster, 2018)

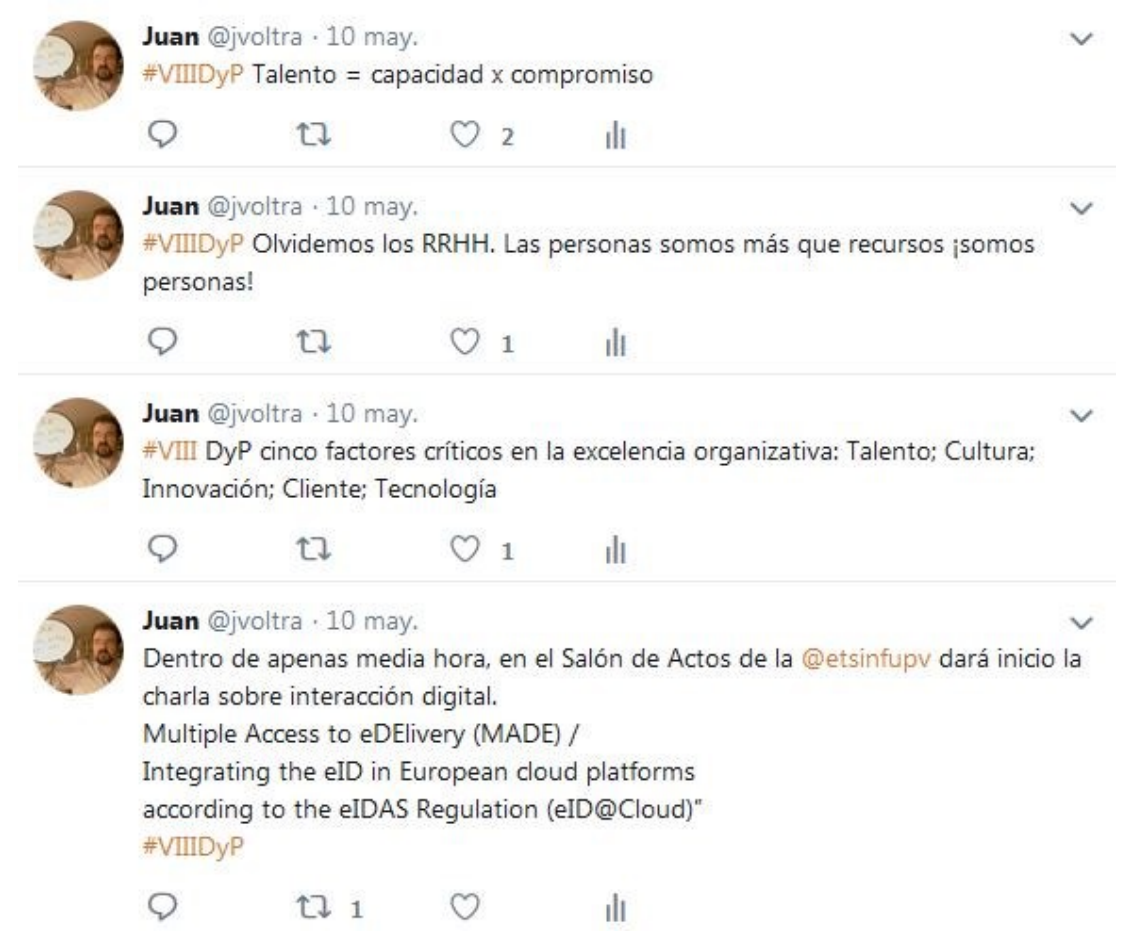

Fig. 2 Tuits iniciales. Elaboración propia.

\section{Resultados}

De los 369 alumnos participaron 287. Como el número excede del aforo del local, es obvio que una parte de ellos accedió al vídeo, bien en el momento de la emisión, bien a posteriori. Un 78\% de participación es un éxito en este tipo de experiencia, recordemos que en los años precedentes, el total de participantes rondaba el 50\% de los matriculados.

Un éxito similar se muestra en las notas obtenidas de la actividad, siendo la media un notable alto, mientras que en ocasiones precedentes tan solo se rozó, en un par de ocasiones, el notable bajo.

Nuestra interpretación es que el interés despertado, tanto de forma previa, como por los contenidos expuestos, ha sido alto. 


\section{Conclusiones}

Los resultados obtenidos nos invitan a profundizar en esta vía. Para el curso siguiente está previsto realizar encuestas mediante una app (Socrative, 2018) durante la charla, tanto de forma presencia como a distancia, a los espectadores de la misma, para poder conducir los contenidos de la misma en el momento de su desarrollo.

Al tiempo, los resultados obtenidos en una experiencia paralela con Twitter, invitan a explotar más esa vía, para lograr un mayor acercamiento al alumno. (Oltra Gutiérrez, Del aula a la realidad inmediata. Uso de noticias para desarrollar la competencia responsabilidad ética, medioambiental y profesional, 2018)

\section{Agradecimientos}

El presente trabajo ha sido desarrollado en el marco del Proyecto "Multiple Access to eDElivery (MADE)" (Ref.: INEA/CEF/ICT/A2016/1278042) dentro del programa "Connecting Europe Facility (CEF) y dentro del sector Telecom, financiado por la Unión Europea a quien los autores agradecen expresamente este apoyo.

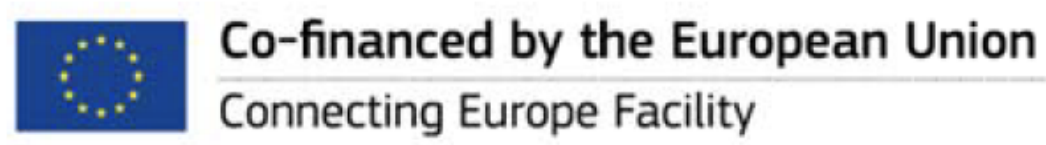

\section{Referencias}

Oltra Gutiérrez, J. V. (2018). Del aula a la realidad inmediata. Uso de noticias para desarrollar la competencia responsabilidad ética, medioambiental y profesional. JDINF 18. Valencia: Universitat Politècnica de València.

Oltra Gutiérrez, J. V. (2018). Gamificación e Informática. Comparativa de una experiencia mixta en Grado y Máster. INRED 18. Valencia: Universitart Politècnica de València.

Oltra Gutiérrez, J. V. (2018). Método del caso como medio de evaluación dentro del modelo FLIP-Teaching. Una experiencia con una asignatura de corte jurídico para ingenieros. INRED 18. Valencia: Ujniversitat Politècnica de Valéncia.

Socrative. (2018). Socrative. Recuperado el 15 de julio de 2018, de https://www.socrative.com/ 
Universidad Carlos III de Madrid. (7 de septiembre de 2017). La UC3M coordina un proyecto para la creación de un espacio común europeo de intercambio electrónico de documentos. Recuperado el 15 de julio de 2018, de NOTICIAS: https://www.uc3m.es/ss/Satellite/UC3MInstitucional/es/Detalle/Comunicacion_C/1371236 508424/1371215537949/La_UC3M_coordina_un_proyecto_para_la_creacion_de_un_espac io_comun_europeo_de_intercambi 